\title{
ON THE HILBERT FUNCTION OF DETERMINANTAL RINGS AND THEIR CANONICAL MODULE
}

\author{
ALDO CONCA AND JÜRGEN HERZOG
}

(Communicated by Wolmer V. Vasconcelos)

\begin{abstract}
We determine the Hilbert function of a determinantal ring and of its canonical module using a combinatorial result of Krattenthaler. This gives a new proof of Abhyankar's formula.
\end{abstract}

Let $K$ be a field and $X=\left(X_{i j}\right)$ be an $m \times n$-matrix of indeterminates, with $m \leq n$. We denote by $K[X]$ the polynomial ring over $K$ in the indeterminates $X_{i j}$ and by $I_{r+1}(X)$ the ideal in $K[X]$ generated by the $r+1$-minors of $X$ and set

$$
R_{r+1}=K[X] / I_{r+1}(X) .
$$

The purpose of this note is to derive a compact formula for the Hilbert series of $R_{r+1}$ and its canonical module. The result is actually a simple rewriting of Abhyankar's formula [1]. However, we want to point out that our formula can as well be obtained from a combinatorial result of Krattenthaler [7] and thus gives a new proof of Abhyankar's formula. Of course, the burden of the proof is hidden in the combinatorial part.

Krattenthaler counts the nonintersecting paths with a given number of corners. To be precise consider the set of points $V=\{(i, j): 1 \leq i \leq m$, $1 \leq j \leq n\}$. We define a partial order on $V$ by setting $(i, j) \leq\left(i^{\prime}, j^{\prime}\right)$ if $i \geq i^{\prime}$ and $j \leq j^{\prime}$. Let $P, Q \in V$; a maximal chain $C$ in $V$ with end points $P$ and $Q$ will be called a path from $P$ to $Q$. A corner of $C$ is an element $(i, j) \in C$ for which $(i-1, j)$ and $(i, j-1)$ belong to $C$ as well. The path in Figure 1 on the next page has two corners.

Let $P_{i}, Q_{i}, i=1, \ldots, r$, be points of $V$. A subset $W \subset V$ is called an $r$-tuple of nonintersecting paths from $P_{i}$ to $Q_{i}(i=1, \ldots, r)$ if $W=$ $C_{1} \cup C_{2} \cup \cdots \cup C_{r}$ where each $C_{i}$ is a path from $P_{i}$ to $Q_{i}$ and where $C_{i} \cap C_{j}=\varnothing$ if $i \neq j$. The number of corners $c(W)$ of $W$ is the sum of the number of corners of the $C_{i}, i=1, \ldots, r$.

Received by the editors February 8, 1993.

1991 Mathematics Subject Classification. Primary 14M12; Secondary 13D40, 13 C40.

The first author's research was supported by a grant from Consiglio Nazionale delle Ricerche, Italy. 


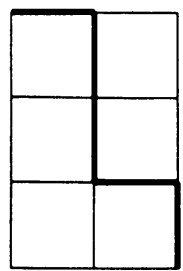

FIGURE 1

Theorem (Kulkarni, Krattenthaler). Let $P_{i}=\left(a_{i}, n\right), 1 \leq a_{1}<\cdots<a_{r} \leq m$, and $Q_{i}=\left(m, b_{i}\right), 1 \leq b_{1}<\cdots<b_{r} \leq n$. Then the number of nonintersecting paths from $P_{i}$ to $Q_{i}, i=1, \ldots, r$, with exactly $k$ corners is given by

$$
\sum \operatorname{det}\left(\left(\begin{array}{c}
m-a_{j}-i+j \\
k_{i}
\end{array}\right)\left(\begin{array}{c}
n-b_{i}+i-j \\
k_{i}+i-j
\end{array}\right)\right)_{i, j=1, \ldots, r},
$$

where the sum is taken over all sequences $\left(k_{1}, \ldots, k_{r}\right)$ such that $\sum_{i=1}^{r} k_{i}=k$.

Kulkarni [8] deduces this theorem from Abhyankar's formula for the Hilbert function of a determinantal ring, while Krattenthaler [7] gives a purely combinatorial proof of it. Actually his result is more general, that is, he allows the end points to be in a more general position.

Let us indicate, as described in [5] and [4], or in Kulkarni's paper [8], how the Hilbert series of determinantal rings is related to the nonintersecting paths. Given integers $1 \leq a_{1}<\cdots<a_{r} \leq m$ and $1 \leq b_{1}<\cdots<b_{r} \leq n$, we denote by $\left[a_{1}, \ldots, a_{r} \mid b_{1}, \ldots, b_{r}\right]$ the minor with the rows $a_{1}, \ldots, a_{r}$ and the columns $b_{1}, \ldots, b_{r}$. The set of all minors $P(X)$ is a poset with the following partial order:

$$
\left[a_{1}, \ldots, a_{r} \mid b_{1}, \ldots, b_{r}\right] \leq\left[c_{1}, \ldots, c_{s} \mid d_{1}, \ldots, d_{s}\right]
$$

if $r \geq s$ and $a_{1} \leq c_{1}, \ldots, a_{s} \leq c_{s}, b_{1} \leq d_{1}, \ldots, b_{s} \leq d_{s}$. Let $\sigma \in P(X)$; then we denote by $I_{\sigma}(X)$ the ideal generated by the minors in the set $\{\eta \in$ $P(X): \eta \nsupseteq \sigma\}$. In case $\sigma=[1, \ldots, r \mid 1, \ldots, r]$ one obtains $I_{\sigma}(X)=I_{r+1}(X)$.

It is shown in [5] that for a suitable term order (order of the monomials) the ideal of initial forms $I_{\sigma}(X)^{*}$ of $I_{\sigma}(X)$ is generated by squarefree monomials. Thus $K[X] / I_{\sigma}(X)^{*}$ may be viewed as a Stanley-Reisner ring of a certain simplicial complex $\Delta_{\sigma}$. Further it is shown in [5] that $\Delta_{\sigma}$ is shellable and that its facets may be identified with the nonintersecting paths connecting $P_{i}=\left(a_{i}, n\right)$ with $Q_{i}=\left(m, b_{i}\right)$ for $i=1, \ldots, r$. Using the fact that $R_{\sigma}=K[X] / I_{\sigma}(X)$ and $K[X] / I_{\sigma}(X)^{*}$ have the same Hilbert series and that $\Delta_{\sigma}$ is shellable, one deduces as in [4] from the McMullen-Walkup formula that the Hilbert series $H_{R_{\sigma}}(t)$ of $R_{\sigma}$ is of the form

$$
H_{R_{\sigma}}(t)=\frac{\sum_{k} h_{k} t^{k}}{(1-t)^{d}}
$$

where $d=\operatorname{dim} R_{\sigma}(X)$ and where $h_{k}$ is the number of the nonintersecting paths from $P_{i}$ to $Q_{i}(i=1, \ldots, r)$ with exactly $k$ corners. Thus applying 
the theorem we get

$$
H_{R_{\sigma}}(t)=\frac{\operatorname{det}\left(\sum_{k}\left(\begin{array}{c}
m-a_{j}-i+j \\
k
\end{array}\right)\left(\begin{array}{c}
n-b_{i}+i-j \\
k+i-j
\end{array}\right) t^{k}\right)_{i, j=1, \ldots, r}}{(1-t)^{d}},
$$

where $d=(m+n+1) r-\sum_{i=1}^{r}\left(a_{i}+b_{i}\right)$ is the dimension of $R_{\sigma}$.

From now on we concentrate our attention to the Hilbert function of the ring $R_{r+1}$ which is defined by the ideal of minors $I_{r+1}(X)$ of size $r+1$. In this case we have to consider the nonintersecting paths from $P_{i}=(i, n)$ to $Q_{i}=(m, i)$. Let us denote by $\mathscr{C}\left(P_{i}, Q_{j}\right)_{k}$ the number of paths from $P_{i}$ to $Q_{j}$ with exactly $k$ corners and by $\mathscr{C}\left(P_{i}, Q_{j}\right)$ the polynomial $\sum_{k} \mathscr{C}\left(P_{i}, Q_{j}\right)_{k} t^{k}$. Then we have Corollary 1.

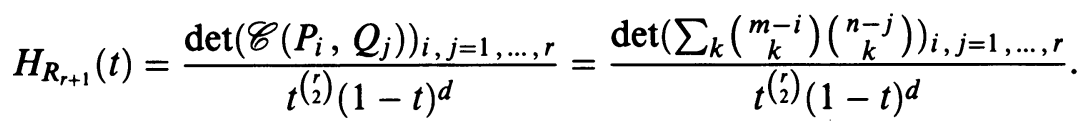

Proof. For $P=(a, b)$ we set $|P|=a^{2}+b^{2}$. One proves easily by induction on $\left|P_{i}-Q_{j}\right|$ that $\mathscr{C}\left(P_{i}, Q_{j}\right)_{k}=\left(\begin{array}{c}m-i \\ k\end{array}\right)\left(\begin{array}{c}n-j \\ k\end{array}\right)$. Thus it remains to show that

$$
\begin{aligned}
& \operatorname{det}\left(\sum_{k}\left(\begin{array}{c}
m-i \\
k
\end{array}\right)\left(\begin{array}{c}
n-j \\
k+i-j
\end{array}\right) t^{k}\right)_{i, j=1, \ldots, r} \\
& =\frac{1}{t^{\left(\begin{array}{r}
2 \\
2
\end{array}\right)}} \operatorname{det}\left(\sum_{k}\left(\begin{array}{c}
m-i \\
k
\end{array}\right)\left(\begin{array}{c}
n-j \\
k
\end{array}\right) t^{k}\right)_{i, j=1, \ldots, r} .
\end{aligned}
$$

This identity is a special case $(u=0)$ of the next

Lemma. Let $u \geq 0$ an integer, and consider the following $r \times r$ matrices of polynomials

$$
\begin{gathered}
H_{u}=\left(\sum_{k}\left(\begin{array}{c}
m-i \\
k
\end{array}\right)\left(\begin{array}{c}
n-j \\
k+u+i-j
\end{array}\right) t^{k}\right), \\
H_{u}^{\prime}=\left(\frac{1}{t^{i-1}} \sum_{k}\left(\begin{array}{c}
m-i \\
k
\end{array}\right)\left(\begin{array}{c}
n-j \\
k+u
\end{array}\right) t^{k}\right), \\
A=\left((-1)^{i-j}\left(\begin{array}{c}
i-1 \\
j-1
\end{array}\right) \frac{1}{t^{i-j}}\right), \quad \text { and } B=\left((-1)^{j-i}\left(\begin{array}{c}
j-1 \\
i-1
\end{array}\right)\right) .
\end{gathered}
$$

Then $H_{u}^{\prime}=A H_{u} B$. In particular, since $A$ and $B$ are triangular matrices whose diagonal elements are all 1 , it follows that $\operatorname{det} H_{u}^{\prime}=\operatorname{det} H_{u}$.

The proof follows by straightforward calculation using the identity

$$
\sum_{q \geq 1}(-1)^{p-q}\left(\begin{array}{l}
p-1 \\
q-1
\end{array}\right)\left(\begin{array}{l}
a-q \\
b-q
\end{array}\right)=(-1)^{p-1}\left(\begin{array}{l}
a-p \\
b-1
\end{array}\right) .
$$

In order to compute the Hilbert series of the graded canonical module $\omega_{r+1}$ of $R_{r+1}$ we use the equation

$$
H_{\omega_{r+1}}(t)=(-1)^{d} H_{R_{r+1}}\left(t^{-1}\right),
$$

where $d=(n+m-r) r$ is the dimension of $R_{r+1}$, and obtain 


\section{Corollary 2.}

$$
\begin{aligned}
H_{\omega_{r+1}}(t) & =\frac{t^{n r} \operatorname{det}\left(\sum_{k}\left(\begin{array}{c}
m-i \\
k
\end{array}\right)\left(\begin{array}{c}
n-j \\
n-m+k+i-j
\end{array}\right) t^{k}\right)}{(1-t)^{d}} \\
& =\frac{t^{n r} \operatorname{det}\left(\sum_{k}\left(\begin{array}{c}
m-i \\
k
\end{array}\right)\left(\begin{array}{c}
n-j \\
n-m+k
\end{array}\right) t^{k}\right)}{t^{(r} \begin{array}{c}
r \\
2
\end{array}(1-t)^{d}} .
\end{aligned}
$$

Proof. The first equation follows directly from the substitution of $t$ by $t^{-1}$, while for the second equation we use our lemma with $u=n-m$.

Recall that, by a result of Stanley, a Cohen-Macaulay domain homogeneous $K$-algebra $R$ is Gorenstein if and only if

$$
H_{R}(t)=(-1)^{d} t^{a} H_{R}\left(t^{-1}\right)
$$

for some $a$. If this is the case, then $a$ is the degree of the rational function $H_{R}(t)$, the so-called $a$-invariant of $R$.

Comparing the formulas in Corollaries 1 and 2 we deduce the well-known fact that $R_{r+1}$ is Gorenstein if and only if $m=n$.

As a last application we compute the Cohen-Macaulay type $r\left(R_{r+1}\right)$ of $R_{r+1}$, that is, the minimal number of generators of $\omega_{r+1}$. For this we use the fact, proved by Bruns [2] (see also [3]), that $R_{r+1}$ is a level ring, which means that all generators of $\omega_{r+1}$ have the same degree. Therefore, by (3) and Corollary 1, the type of $R_{r+1}$ is the leading coefficient of the polynomial $\operatorname{det}\left(\sum_{k}\left(\begin{array}{c}m-i \\ k\end{array}\right)\left(\begin{array}{c}n-j \\ k\end{array}\right) t^{k}\right)$. The $(i, j)$ th polynomial in the matrix has degree $m-i$ for $j \leq n-m+i$ and degree $n-j$ for $j>n-m+i$. Hence we see that

$$
r\left(R_{r+1}\right)=\operatorname{det}\left(\left(\begin{array}{c}
n-j \\
m-i
\end{array}\right)\right)_{i, j=1, \ldots, r} .
$$

Using the Vandermonde determinant we get

$$
r\left(R_{r+1}\right)=\prod_{i=1}^{r}\left(\begin{array}{c}
n-i \\
m-r
\end{array}\right) /\left(\begin{array}{c}
m-i \\
m-r
\end{array}\right) .
$$

In [3] Bruns and Vetter quote the formula $r\left(R_{r+1}\right)=\prod_{i=1}^{m-r}\left(\begin{array}{c}n-i \\ r\end{array}\right) /\left(\begin{array}{c}m-i \\ r\end{array}\right)$. This formula was obtained by J. Brennan from the explicit computation of the Hilbert series of Schubert varieties due to Hodge and Pedoe [6, Theorem 3, p. 387]. It can be shown directly, by induction on $r$, that these formulas agree. In particular, one sees that

$$
r\left(R_{r+1}\right)=r\left(R_{m-r+1}\right)
$$

We conclude with one observation. The formula in Corollary 1 has the following combinatorial interpretation. Let $\sigma$ be an element of $S_{r}$, the group of permutations of $r$ elements. Let us denote by $\mathscr{C}\left(P_{\sigma}, Q\right)_{k}$ the number of the families of paths from $P_{\sigma(i)}=(\sigma(i), n)$ to $Q_{i}=(m, i), i=1, \ldots, r$, with $k$ corners, and by $\mathscr{C}(P, Q)_{k}^{+}$the number of the nonintersecting paths from $(i, n)$ to $(m, i), i=1, \ldots, r$, with $k$ corners. Expanding the determinant 


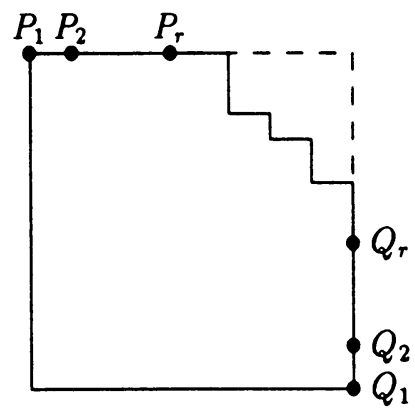

FigURE 2

in the formula in Corollary 1 , one gets

$$
\mathscr{C}(P, Q)_{k-\left(\begin{array}{l}
r \\
2
\end{array}\right)}^{+}=\sum_{\sigma \in S_{r}}(-1)^{\sigma} \mathscr{C}\left(P_{\sigma}, Q\right)_{k}
$$

for all $k \geq 0$. It would be nice to have a direct proof of this identity. We believe that it is true even when we consider paths in a restricted region having the shape of a one-sided ladder, as indicated in Figure 2. We can give a proof for the case when $r=2$.

\section{REFERENCES}

1. S. S. Abhyankar, Enumerative combinatorics of Young tableaux, Marcel Dekker, New York, 1988.

2. W. Bruns, The canonical module of a determinantal ring, Commutative Algebra, Durham 1981 (R. Y. Sharp, ed.), London Math. Soc. Lecture Note Ser., vol. 72, Cambridge Univ. Press, Cambridge, 1982, pp. 109-120.

3. W. Bruns and U. Vetter, Determinantal rings, Lecture Notes in Math., vol. 1327, Springer, Heidelberg, 1988.

4. W. Bruns and J. Herzog, On the computation of a-invariants, Manuscripta Math. 77 (1992), 201-213.

5. J. Herzog and N. V. Trung, Gröbner bases and multiplicity of determinantal and pfaffian ideals, Adv. Math. 96 (1992), 1-37.

6. W. V. D. Hodge and D. Pedoe, Methods of algebraic geometry, Vol. II, Cambridge Univ. Press, Cambridge, 1952.

7. C. Krattenthaler, Non-Crossing two-rowed arrays and summations for Schur functions, preprint, 1992.

8. D. M. Kulkarni, Counting of paths and coefficients of Hilbert polynomial of a determinantal ideal, preprint.

FB6 Mathematik und INFormatik, Universität Gesamthochschule EsSen, UniversiTÄTSSTR. 3, 45117 ESSEN, GERMANY

E-mail address: mat 3020vm.hrz.uni-essen.de

E-mail address: mat $3000 \mathrm{vm} \cdot \mathrm{hrz}$.uni-essen.de 